\title{
Numerically consistent regularization of force-based frame elements
}

\author{
M. H. Scott*, $\dagger$ and O. M. Hamutçuoğlu \\ School of Civil and Construction Engineering, Oregon State University, Corvallis, OR 97331, U.S.A.
}

\begin{abstract}
SUMMARY
Recent advances in the literature regularize the strain-softening response of force-based frame elements by either modifying the constitutive parameters or scaling selected integration weights. Although the former case maintains numerical accuracy for strain-hardening behavior, the regularization requires a tight coupling of the element constitutive properties and the numerical integration method. In the latter case, objectivity is maintained for strain-softening problems; however, there is a lack of convergence for strain-hardening response. To resolve the dichotomy between strain-hardening and strain-softening solutions, a numerically consistent regularization technique is developed for force-based frame elements using interpolatory quadrature with two integration points of prescribed characteristic lengths at the element ends. Owing to manipulation of the integration weights at the element ends, the solution of a Vandermonde system of equations ensures numerical accuracy in the linear-elastic range of response. Comparison of closed-form solutions and published experimental results of reinforced concrete columns demonstrates the effect of the regularization approach on simulating the response of structural members. Copyright (C) 2008 John Wiley \& Sons, Ltd.
\end{abstract}

Received 7 January 2008; Revised 28 March 2008; Accepted 13 April 2008

KEY WORDS: structures; frame analysis; numerical integration; damage; regularization

\section{INTRODUCTION}

Simulating localized response of structural systems using strain-softening constitutive models poses several computational challenges since the equilibrium solution becomes ill-posed and the results are mesh-dependent. This is of importance when evaluating the resistance of a structure to extreme loadings, such as earthquakes. In the analysis of localized response in structural members modeled by continuum finite elements, Bažant and Oh [1] propose a crack band theory where the material

\footnotetext{
${ }^{*}$ Correspondence to: M. H. Scott, School of Civil and Construction Engineering, Oregon State University, 220 Owen Hall, Corvallis, OR 97331, U.S.A.

†E-mail: michael.scott@oregonstate.edu

Contract/grant sponsor: Oregon Department of Transportation (ODOT)
}

Copyright (C) 2008 John Wiley \& Sons, Ltd. 
behavior is defined by parameters of fracture energy, uniaxial strength limit and crack bandwidth. Further advances in the continuum analysis of strain-softening behavior analysis by de Borst and Muhlhaus [2] led to plasticity theory based on higher-order spatial gradients of plastic strains. Simo et al. [3] developed a regularized discontinuous finite element method to overcome problems with mesh dependence in conventional methods. Wells et al. [4] present a regularized mesh-independent continuum method using discontinuous displacement functions in a strain-softening medium.

Consistent with findings in the continuum context, finite element formulations of frame elements exhibit similar problems of ill-posedness and mesh dependence in the presence of strain-softening $[5,6]$. Several approaches to simulating the localized response of frame members have been proposed in the literature. Early attempts to model such behavior used non-linear moment-rotation springs concentrated at the ends of a linear-elastic element $[7,8]$. This approach has been extended in more recent papers and the references therein to include axial-moment interaction $[9,10]$, phenomenological hysteretic models of strength and stiffness degradation [11,12], as well as strain-softening behavior [13,14]. Although this approach is computationally efficient, it generally requires a calibration of the element properties based on the loading conditions and constitutive properties. More recent approaches use strong discontinuities in the beam displacement field to effectively incorporate local dissipative mechanisms in a general finite element setting [15].

Distributed plasticity formulations of frame finite elements offer a more flexible modeling approach to concentrated plasticity by uncoupling the element state determination procedure from the constitutive properties. Several formulations of distributed plasticity are available and fall into three main categories: displacement-based, force-based and mixed [16-18]. Comparisons by Hjelmstad and Taciroglu [19] show that there is no clear winner among the three formulations; however, each has distinct advantages. In the case of force-based elements [20-22], the primary advantage is they satisfy equilibrium in strong form, even in the range of non-linear material response. This allows an analyst to use a coarse finite element mesh in simulating material nonlinear frame response under small displacements, and convergence is achieved by increasing the number of integration points rather than by mesh refinement.

Unlike displacement-based formulations where localization occurs over the length of an entire element, strain-softening behavior causes deformations to localize at a single integration point in a force-based element. To establish objective response, regularization methods have been proposed in the literature for force-based elements. Coleman and Spacone [23] present a regularization method based on a constant fracture energy release to maintain objective response. However, this approach requires an analyst to modify the material properties based on the number of GaussLobatto integration points and a prescribed characteristic length. Subsequent work by Addessi and Ciampi [24] and Scott and Fenves [25] regularize force-based element response by scaling integration weights at the element ends to match prescribed characteristic lengths. Although this approach ensures regularized response for strain-softening behavior without modifying the element constitutive properties, the response is too flexible when these integration methods are used to simulate strain-hardening response. As a result, an analyst must know a priori whether to use a standard or a regularized integration approach when the answer may not be obvious from the given material properties and loading conditions.

An integration approach is developed in this paper to allow an analyst to regularize force-based element response while maintaining numerical accuracy for strain-hardening behavior. The paper starts with basic formulations of force-based frame elements followed by a summary of existing regularization techniques based on the modification of integration weights. Alternative regularization methods based on interpolatory quadrature are explored in order to arrive at the proposed 
method. Examples with the new method are compared with closed-form solutions to confirm the numerical accuracy for strain-hardening behavior. For strain-softening behavior requiring regularization, numerical solutions are compared with published test results of cyclically loaded reinforced concrete columns.

\section{FORCE-BASED ELEMENT FORMULATION}

The force-based element formulation consists of interpolation of basic forces within a basic system settled on the principle of small deformations [22]. Vectors $\mathbf{q}$ and $\mathbf{v}$ represent forces and deformations, respectively, of a corotating frame of reference, or basic system, for the element [26], as shown in Figure 1. Thus, the developments described in this paper are applicable to the large displacement analysis of frames using the corotational formulation [27].

The sectional forces are defined by end forces and interpolation functions. At the element level, equilibrium is stated in the form

$$
\mathbf{s}(x)=\mathbf{b}(x) \mathbf{q}+\mathbf{s}_{p}(x)
$$

where the section forces are in the vector $\mathbf{s}(x)=[N(x) M(x)]^{\mathrm{T}}$. Member loads are not considered in this paper; therefore, the particular equilibrium solution, $\mathbf{s}_{p}(x)$, is equal to zero. The matrix $\mathbf{b}$ contains interpolation functions for the section forces in terms of the basic end forces

$$
\mathbf{b}(x)=\left[\begin{array}{ccc}
1 & 0 & 0 \\
0 & x / L-1 & x / L
\end{array}\right]
$$

From the principle of virtual forces, the element compatibility relation is formulated and the element deformations, $\mathbf{v}$, are obtained in terms of section deformations, e, along the element. For non-linear material response, the compatibility relationship is approximated by numerical integration over $N$ discrete points

$$
\mathbf{v}=\sum_{j=1}^{N} \mathbf{b}_{j}^{\mathrm{T}} \mathbf{e}_{j} w_{j}
$$

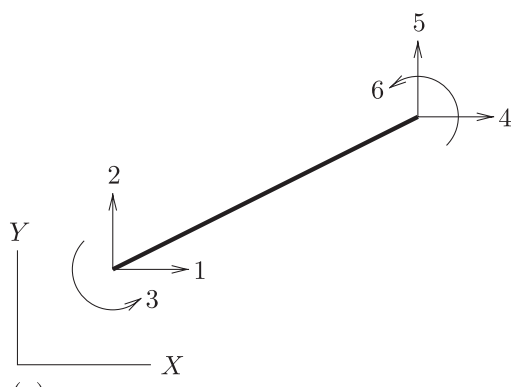

(a)

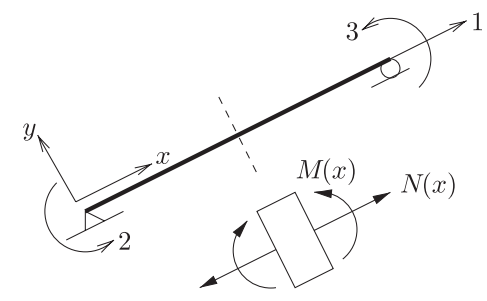

(b)

Figure 1. Degrees of freedom for plane frame elements: (a) forces and displacements in a global coordinate system and (b) forces and deformations in a basic system. 
where $\mathbf{b}_{j}$ and $\mathbf{e}_{j}$ represent the interpolation function and section deformation, respectively, evaluated at the $j$ th integration point location $x_{j}$ with associated weight $w_{j}$. The derivative of the compatibility equation with respect to basic force vector provides the element flexibility matrix, $\mathbf{f}$, in terms of section flexibility

$$
\mathbf{f}=\frac{\partial \mathbf{v}}{\partial \mathbf{q}}=\sum_{j=1}^{N} \mathbf{b}_{j}^{\mathrm{T}} \mathbf{f}_{s j} \mathbf{b}_{j} w_{j}
$$

Neuenhofer and Filippou [28] provide full details of the force-based element formulation in a standard stiffness-based finite element setting, while the variational basis for such elements is described by Hjelmstad and Taciroglu [29]. Extensions of the formulation to include section shear effects are given by Ranzo and Petrangeli [30], Schulz and Filippou [31] and Marini and Spacone [32], while the extension to large deformations is described by De Souza [33].

The most common integration approach to evaluate Equations (3) and (4) is Gauss-Lobatto quadrature [34], which places sample points at the element ends where bending moments are largest in the absence of member loads. The order of accuracy, i.e. the highest monomial integrated exactly, for Gauss-Lobatto quadrature is $2 N-3$. Thus, to obtain the exact solution for a linearelastic, prismatic frame element, e.g. during a patch test [35], at least three Gauss-Lobatto points are required since quadratic polynomials appear in the integrand of Equation (3) in this case. A unique solution is obtained for strain-hardening problems by increasing the number of integration points in a single force-based element. Four to six points are typically sufficient to represent the spread of plasticity along an element [28].

In the presence of strain-softening section response where deformations localize at a single integration point, the solution depends on the characteristic length implied by the Gauss-Lobatto integration weights. This leads to a loss of objectivity since the force-based element response will change as a function of the number of integration points selected by the analyst. Coleman and Spacone [23] regularize the element response using a criterion of constant energy release based on the number of Gauss-Lobatto points and a characteristic length. The advantage of this approach is it does not alter the integration weights of the Gauss-Lobatto rule and thus maintains numerical accuracy for strain-hardening response. However, the main drawback is the regularization ties the section material model to the element integration method, leading to a loss of objectivity of the section response.

\section{REGULARIZATION BASED ON SCALING INTEGRATION WEIGHTS}

An alternative force-based element regularization method is to scale the element integration weights to match prescribed characteristic lengths. This approach is based on dividing an element into three regions (one plastic hinge region at each end and one interior region) then applying separate integration rules over each region.

Addessi and Ciampi [24] use Gauss-Lobatto integration over each region, e.g. a two-point rule over the plastic hinge regions and a three-point rule over the interior. To regularize the element response, the integration rules over the plastic hinge regions are scaled by a factor of 2 in order to make the integration weights at the element ends equal to the characteristic lengths, $l_{p I}$ and $l_{p J}$, specified by the analyst. As shown in Figure 2(a), the integration point locations are

$$
x=\left\{0,2 l_{p I}, 2 l_{p I}, L_{\mathrm{int}} / 2, L-2 l_{p J}, L-2 l_{p J}, L\right\}
$$




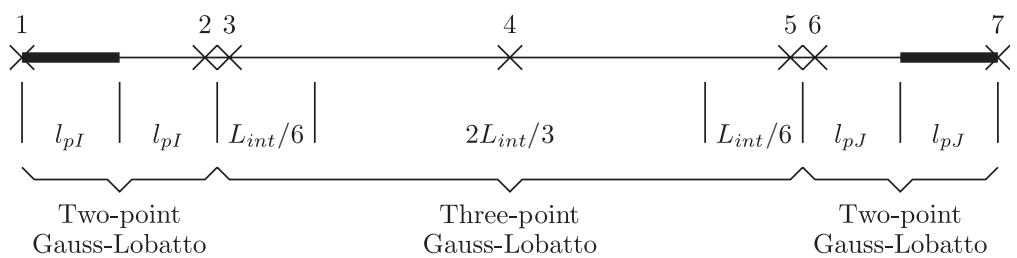

(a)

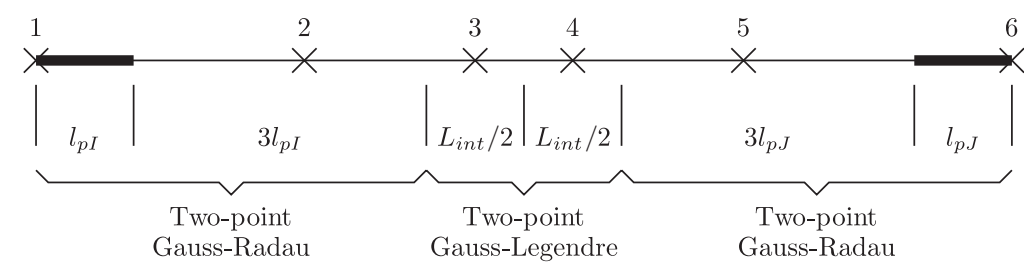

(b)

Figure 2. Force-based element regularization methods based on scaling integration weights in the plastic hinge regions: (a) Gauss-Lobatto over the plastic hinge and interior regions and (b) Gauss-Radau in the plastic hinge regions and Gauss-Legendre over the interior.

and the associated weights are

$$
w=\left\{l_{p I}, l_{p I}, L_{\mathrm{int}} / 6,2 L_{\mathrm{int}} / 3, L_{\mathrm{int}} / 6, l_{p J}, l_{p J}\right\}
$$

where $L_{\text {int }}=L-2 l_{p I}-2 l_{p J}$ is the length of the element interior. It is noted that the coincident Gauss-Lobatto integration points in Equation (5), and their corresponding weights in Equation (6), at the interfaces between the plastic hinge regions and the element interior can be combined in order to reduce the number of sample points. Addessi and Ciampi [24] also propose three-point Gauss-Lobatto integration over the plastic hinge regions, in which case quadratic polynomials are represented exactly over the entire element length.

Scott and Fenves [25] apply two-point Gauss-Radau quadrature [34] over the plastic hinge regions and scale the integration weights by 4 in order to regularize the element response. In this case, the length of the element interior is $L_{\text {int }}=L-4 l_{p I}-4 l_{p J}$, over which two-point GaussLegendre quadrature is applied, giving the following integration point locations:

$$
x=\left\{0,8 l_{p I} / 3, x_{3}, x_{4}, L-8 l_{p J} / 3, L\right\}
$$

where $x_{3(4)}=4 l_{p I}+L_{\text {int }}( \pm 1 / \sqrt{3}+1) / 2$. The associated weights are

$$
w=\left\{l_{p I}, 3 l_{p I}, L_{\mathrm{int}} / 2, L_{\mathrm{int}} / 2,3 l_{p J}, l_{p J}\right\}
$$

The mixture of Gauss-Radau and Gauss-Legendre quadrature ensures a sufficient level of integration accuracy while placing sample points at the element ends. The locations and weights of the integration points for this approach are shown in Figure 2(b).

The numerical behavior of regularization methods based on scaling integration weights is demonstrated via the moment-rotation response of a simply supported beam under anti-symmetric bending. As shown in Figure 3, the section moment-curvature relationship is bilinear with hardening ratio $\alpha$. To investigate strain-hardening section behavior, $\alpha$ is set equal to 0.02 , while this 


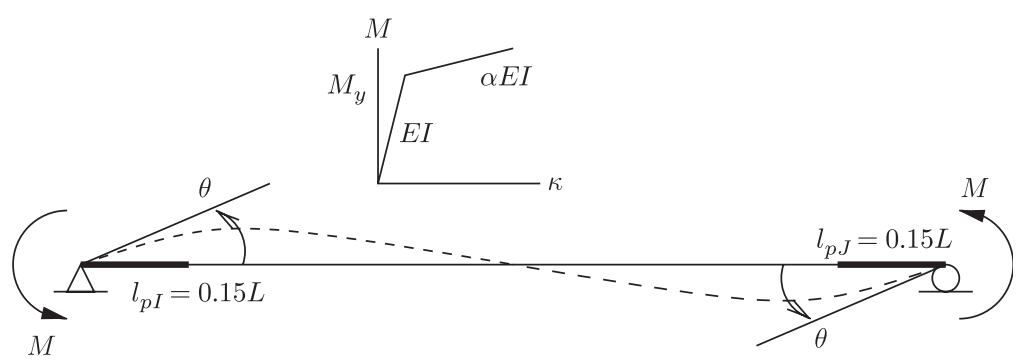

Figure 3. Simply supported beam in a state of anti-symmetric bending and with a bilinear moment-curvature relationship.
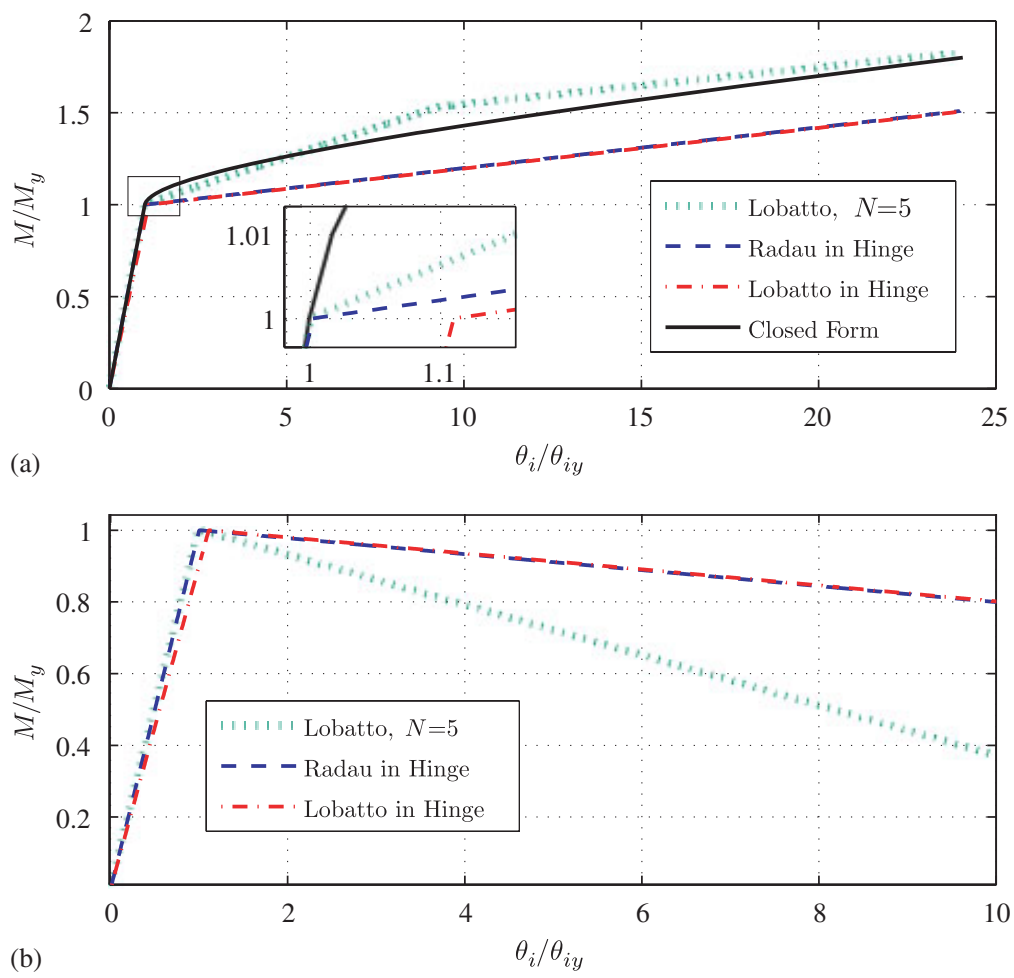

Figure 4. Computed moment-rotation relationship of regularization methods based on scaling integration weights compared with standard five-point Gauss-Lobatto rule

for: (a) strain-hardening behavior and (b) strain-softening section behavior.

parameter is set to -0.02 in order to produce localized response at the element ends. The characteristic plastic hinge lengths are $l_{p I}=l_{p J}=0.15 \mathrm{~L}$.

The solutions obtained by using the integration points and weights in Equations (5)-(6) and (7)-(8) are shown in Figure 4 and compared with that obtained for a standard five-point GaussLobatto rule applied over the element length. For strain-softening behavior that causes localization at the element ends, both regularized integration methods unload at an identical rate, as shown in 
Figure 4(b). The beam unloads at a higher rate for five-point Gauss-Lobatto integration since the implied characteristic length is $0.05 \mathrm{~L}$. On the other hand, five-point Gauss-Lobatto integration gives the best solution for strain-hardening behavior (Figure 4(a)), while the post-yield response of the regularized methods is too flexible compared with the exact solution. This example demonstrates the need to find a single integration method that can accommodate both strain-softening and strainhardening behaviors. To arrive at such a solution, it is worth turning attention to a regularization approach based on interpolatory quadrature.

\section{REGULARIZATION BASED ON INTERPOLATORY QUADRATURE}

An equivalent approach to regularize the force-based element response is to set the integration weights at the element ends equal to characteristic values and then solve a system of equations for the remaining integration point locations and weights to ensure numerical accuracy. In the case where all integration point locations and weights are unknown over the interval $[a, b]$ except for end points of weights $l_{p I}$ and $l_{p J}$, there are $2 N-4$ unknown locations and to weights of the $N-2$ integration points. These unknowns can be found by solving the following system of equations:

$$
\sum_{i=2}^{N-1} x_{i}^{j} w_{i}+a^{j} l_{p I}+b^{j} l_{p J}-\frac{b^{j+1}-a^{j+1}}{j+1}=0, \quad j=0,1, \ldots, 2 N-5
$$

The most common choice to solve Equation (9) is Newton's method [36]; however, its convergence is highly dependent on the initial guess for the unknown locations and weights. The resulting quadrature rule has an order of accuracy of $2 \mathrm{~N}-5$; thus, at least four integration points (two interior points in addition to the two end points) are required to ensure that the element passes a patch test. In the absence of constraints on the end weights, the solution to Equation (9) gives the GaussLobatto locations and weights with accuracy $2 N-3$, while in the absence of any constraints on the locations and weights of the integration points, the solution gives Gauss-Legendre quadrature of accuracy $2 N-1$.

Interpolatory quadrature, where the locations of all the integration points are fixed, gives a more stable solution procedure, albeit with a lower order of accuracy. Specifying all $N$ integration point locations, in addition to setting the integration weights at the element ends to $l_{p I}$ and $l_{p J}$, reduces the order of accuracy to $N-3$ and turns Equation (9) into a linear system of $N-2$ equations for the unknown weights

$$
\begin{aligned}
& {\left[\begin{array}{cccc}
1 & 1 & \cdots & 1 \\
x_{2} & x_{3} & \cdots & x_{N-1} \\
\vdots & \vdots & & \vdots \\
x_{2}^{N-3} & x_{3}^{N-3} & \cdots & x_{N-1}^{N-3}
\end{array}\right]\left[\begin{array}{c}
w_{2} \\
w_{3} \\
\vdots \\
w_{N-1}
\end{array}\right]} \\
& =\left[\begin{array}{c}
b-a-l_{p I}-l_{p J} \\
\left(b^{2}-a^{2}\right) / 2-a l_{p I}-b l_{p J} \\
\vdots \\
\left(b^{N-2}-a^{N-2}\right) /(N-2)-a^{N-3} l_{p I}-b^{N-3} l_{p J}
\end{array}\right]
\end{aligned}
$$


To integrate quadratic polynomials exactly, at least five integration points (three interior points plus two end points) must be used. To prevent poor conditioning of the Vandermonde matrix in Equation (10), the integration point locations should be well spaced and symmetric on the interval of integration and the order of integration, $N$, should be kept low [37]. Better conditioning for interpolatory quadrature rules can be obtained using least squares theory [38].

Although the solutions provided by Equations (9) and (10) regularize the element response for strain-softening section behavior, they suffer from the same shortcomings for strain-hardening behavior as the methods based on scaling integration weights and will thus lead to the same results shown in Figure 4. A further modification is required in order for a single integration method to provide regularized response while maintaining a convergent solution for strain-hardening behavior.

\section{PROPOSED REGULARIZATION METHOD}

As seen in the foregoing discussion, regularizing force-based element response for strain-softening behavior comes at the price of losing numerical accuracy when simulating strain-hardening behavior. This forces an analyst to decide a priori which integration method to use when modeling frame structures with force-based finite elements. For simulations such as reinforced concrete columns with heavy axial loads using fiber models, the answer may not be clear.

To avoid complicated phenomenological rules that couple the integration rule to the section constitutive model, a standard quadrature rule is modified with two additional integration points. These points are placed at small distances, $\xi_{I}$ and $\xi_{J}$, from the element ends, as shown in Figure 5(b):

$$
x=\left\{\left(x_{1}=0\right), \xi_{I}, x_{2}, \ldots, x_{N-1}, L-\xi_{J},\left(x_{N}=L\right)\right\}
$$

From this juxtaposition of integration points, the element response is regularized by setting the weights of the integration points at the element ends equal to $l_{p I}$ and $l_{p J}$, as was the case in the

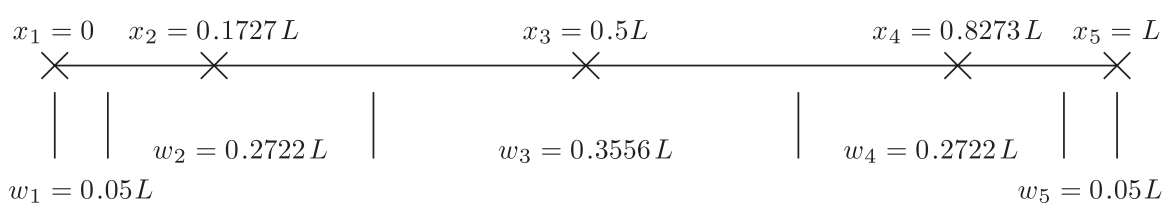

(a)

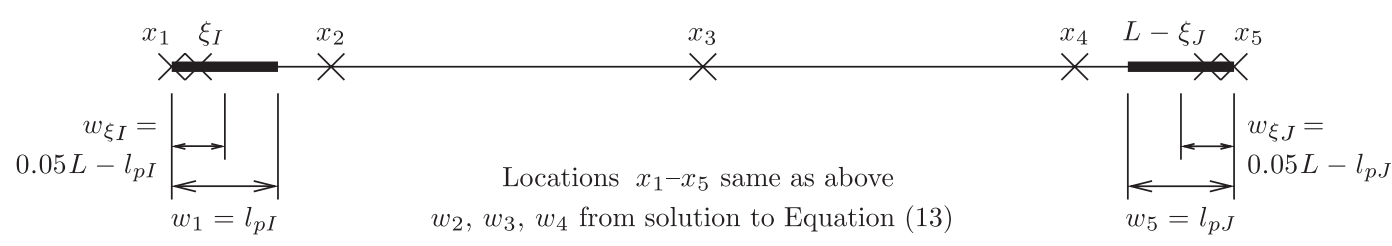

(b)

Figure 5. (a) Standard five-point Gauss-Lobatto integration rule and (b) five-point Gauss-Lobatto rule regularized by addition of two integration points just inside the element ends. 
previous regularization methods. Then, the weights of the integration points at $\xi_{I}$ and $L-\xi_{J}$ are set equal to $w_{1}-l_{p I}$ and $w_{N}-l_{p J}$, respectively, where $w_{1}$ and $w_{N}$ are the end weights of the standard quadrature rule:

$$
w=\left\{l_{p I}, w_{1}-l_{p I}, w_{2}, \ldots, w_{N-1}, w_{N}-l_{p J}, l_{p J}\right\}
$$

This arrangement of integration points at the element ends, demonstrated in Figure 5(b) for a standard five-point Gauss-Lobatto rule, ensures that a convergent strain-hardening solution is maintained; however, manipulating the locations and weights of the integration points will compromise the accuracy of the underlying Gauss-Lobatto quadrature rule. Only constant polynomials can be represented exactly since the sum of the integration weights in Equation (12) remains equal to the element length. For frame analysis, however, quadratic polynomials must be represented exactly in order to capture the exact solution for a linear-elastic, prismatic element. To this end, the integration weights of the element interior can be re-computed using interpolatory quadrature in order to ensure a sufficient level of accuracy for structural engineering applications:

$$
\begin{aligned}
& {\left[\begin{array}{cccc}
1 & 1 & \cdots & 1 \\
x_{2} & x_{3} & \cdots & x_{N-1} \\
\vdots & \vdots & & \vdots \\
x_{2}^{N-3} & x_{3}^{N-3} & \cdots & x_{N-1}^{N-3}
\end{array}\right]\left[\begin{array}{c}
w_{2} \\
w_{3} \\
\vdots \\
w_{N-1}
\end{array}\right]} \\
& =\left[\begin{array}{c}
L-w_{1}-w_{N} \\
L^{2} / 2-L l_{p J}-\xi_{I}\left(w_{1}-l_{p I}\right)-\left(L-\xi_{J}\right)\left(w_{N}-l_{p J}\right) \\
\vdots \\
\frac{L^{N-2}}{N-2}-L^{N-3} l_{p J}-\xi_{I}^{N-3}\left(w_{1}-l_{p I}\right)-\left(L-\xi_{J}\right)^{N-3}\left(w_{N}-l_{p J}\right)
\end{array}\right]
\end{aligned}
$$

For an underlying $N$-point Gauss-Lobatto rule, the order of accuracy will be reduced from $2 N-3$ to $N-3$ after re-computing the integration weights. As a result, there must be at least five integration points of the underlying quadrature method in order for the regularized integration rule to capture the exact solution for a linear-elastic, prismatic element. The underlying quadrature method can provide as few as three integration points while maintaining the exact linear-elastic solution if the constraints on $\left(w_{1}-l_{p I}\right)$ and $\left(w_{N}-l_{p J}\right)$ are removed; however, using this few integration points will lead to a poor representation of the spread of plasticity in strain-hardening problems.

It is emphasized that the proposed regularization method is not restricted to an underlying Gauss-Lobatto quadrature rule. Any $N$ point quadrature method can be used, including NewtonCotes, which spaces integration points equally along the element [34]. In fact, $N$ arbitrarily located integration points can be used; however, this may lead to ill-conditioning of the Vandermonde equations used for interpolatory quadrature. The only restriction is that the underlying quadrature rule places integration points at the element ends. 


\subsection{Condition number}

An additional consideration in constructing numerical integration rules is the condition number $[39,40]$, which is the sum of the absolute values of the integration weights:

$$
K=\sum_{i=1}^{N}\left|w_{i}\right|
$$

For beam-column elements, a well-conditioned quadrature rule has a condition number equal to the element length, $L$. However, for the regularized integration method described in this paper, negative integration weights will appear when $l_{p I}>w_{1}$ or $l_{p J}>w_{N}$. When there are negative weights at each end of the element, it can be shown that the condition number of the regularized integration method is

$$
K=2\left(l_{p I}-w_{1}\right)+2\left(l_{p J}-w_{N}\right)+\tilde{K}
$$

where $\tilde{K}$ is the condition number of the underlying quadrature rule. For values of $l_{p I}$ and $l_{p J}$ that satisfy $l_{p I}+l_{p J}<L$, the condition number of the proposed regularization method remains bounded

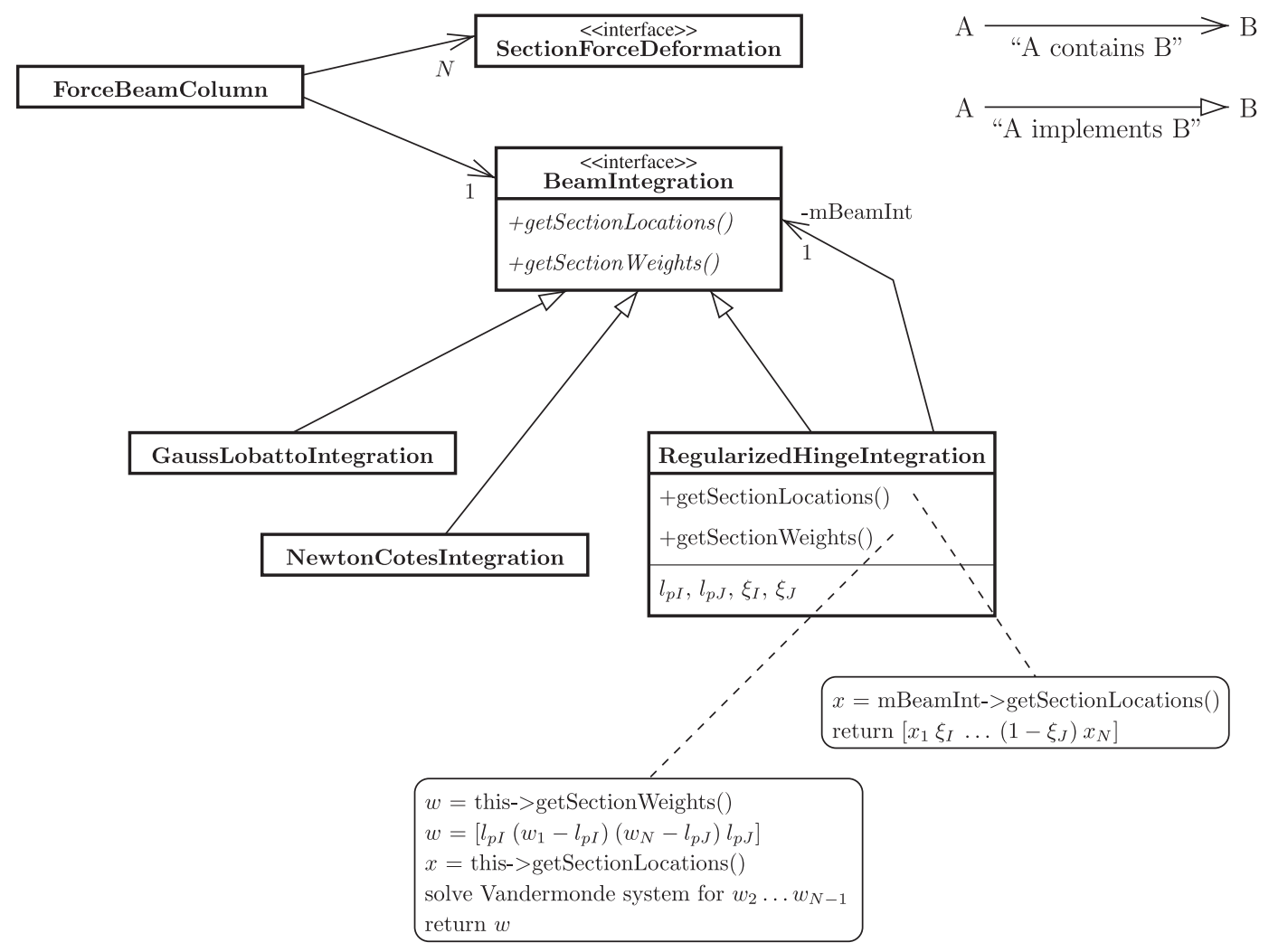

Figure 6. UML diagram for implementation of proposed regularization method in an object-oriented finite element framework. 
by $2 L+\tilde{K}$. Thus, for an underlying quadrature rule that is well conditioned, which is guaranteed for Gauss-Lobatto quadrature with any value of $N$ and Newton-Cotes quadrature for any $N$ except odd values greater than or equal to 9 [34], the proposed method will be numerically stable.

\subsection{Software implementation}

To emphasize the loose coupling of the proposed regularization method from both the underlying quadrature method and the element constitutive behavior, a UML diagram [41] of its implementation in an object-oriented finite element framework is shown in Figure 6. The force-based frame element state determination is encapsulated in a class that contains $N$ instances of a section forcedeformation object and one instance of a beam integration object. This implementation follows the Strategy design pattern of offering an object interchangeable algorithms to define its behavior $[42,43]$. The proposed regularization method is implemented by recursive composition of an object of the same type but different class, e.g. using an implementation of a Gauss-Lobatto or NewtonCotes quadrature rule. The encapsulation of the regularized integration method in an object separate from the element makes the proposed method applicable to the wide range of force-based element state determination algorithms available in the literature [22, 28, 44, 45].

\subsection{Verification example}

To verify that the proposed regularization method is mathematically correct, the response of the simply supported beam in Figure 3 is demonstrated with a five-point Gauss-Lobatto rule regularized with parameters $\xi_{I}=\xi_{J}=0.001 L$ and $l_{p I}=l_{p J}=0.15 L$. The location and weight of the regularized integration points are given in Figure 5(b). After the solution to Equation (13), the interior integration weights are $w_{2}=w_{4}=0.2718 L$ and $w_{3}=0.3563 L$, which differ only slightly from the corresponding weights of the underlying five-point Gauss-Lobatto rule, $w_{2}=w_{4}=0.2722 L$ and $w_{3}=0.3556 L$. This difference increases with increasing values of $\xi_{I}$ and $\xi_{J}$ and would be zero when these parameters are zero; however, this change in integration weights is essential to ensure that the element response is correct in the linear-elastic range of response.

The moment-rotation response with the standard and regularized five-point Gauss-Lobatto integration is presented in Figure 7 for strain-hardening section behavior. As shown in Figure 7(a), there is a slight difference between the response computed with the regularized and nonregularized integration rules. This discrepancy occurs as yielding spreads over distances $\xi_{I}$ and $\xi_{J}$ at the element ends. After these integration points plastify, the regularized solution returns to that obtained by the standard Gauss-Lobatto rule. Thus, the proposed regularization method maintains a convergent solution for strain-hardening problems. This was not possible using the previous regularization methods $[24,25]$.

For the case of localization at the element ends due to strain-softening section behavior, Figure 8(a) shows the regularized five-point Gauss-Lobatto rule unloads at an identical rate to the solution obtained by scaling Gauss-Radau integration weights in the plastic hinge regions [25]. In addition, the response of the standard five-point Gauss-Lobatto rule is repeated in Figure 8(a) for comparison. This idealized example shows that the proposed regularization method is suitable for simulating both strain-hardening and strain-softening section responses. Comparisons with published experimental data in the following section show that the method is applicable to simulating the response of reinforced concrete structural members. 


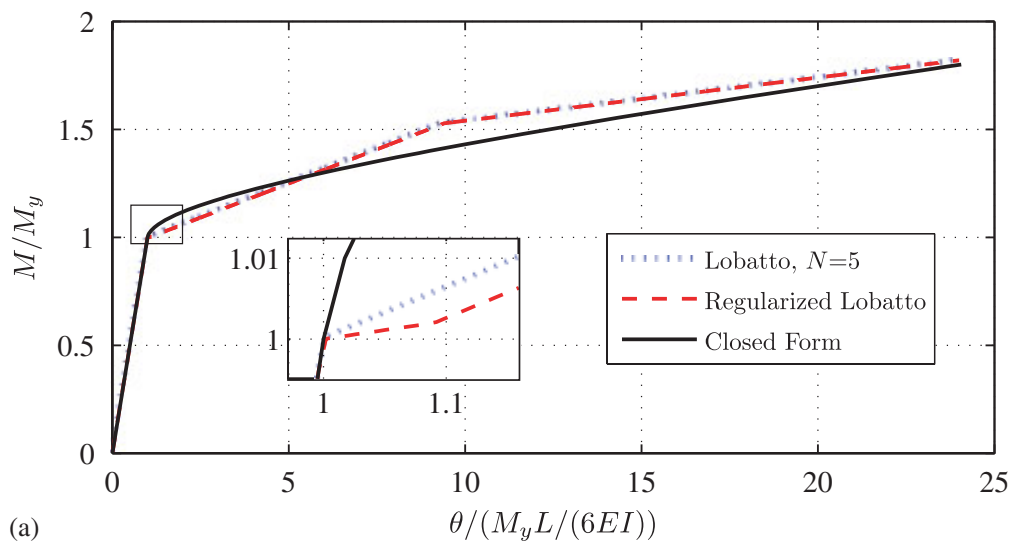

(a)

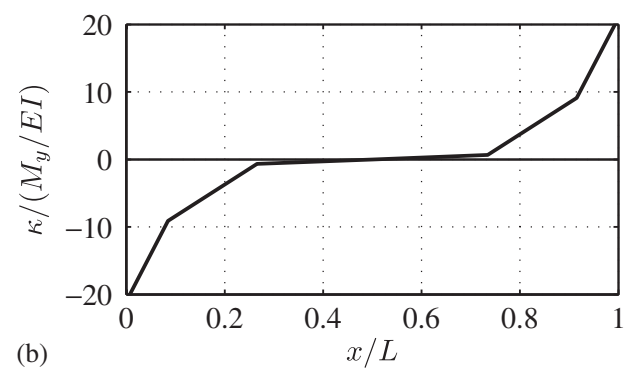

Figure 7. Comparison of beam response between standard and regularized five-point Gauss-Lobatto for strain-hardening section behavior: (a) computed moment-rotation response and (b) curvature distribution along the beam.

\subsection{Sensitivity of $\xi$ parameters}

The most significant source of uncertainty in the proposed regularization method is the values of parameters $\xi_{I}$ and $\xi_{J}$. For strain-hardening problems, these parameters should be relatively small in order to maintain the convergent behavior offered by the underlying quadrature method. The effect of increasing $\xi_{I}$ and $\xi_{J}$ for the strain-hardening example is demonstrated in Figure 9. The computed solution deviates slightly from the standard Gauss-Lobatto solution when $\xi_{I}=\xi_{J}=$ $0.01 L$; however, there is a large error for $\xi_{I}=\xi_{J}=0.1 L$, which places the additional integration points outside the $0.05 \mathrm{~L}$ region associated with the end points of the underlying five-point GaussLobatto rule.

On the other hand, for strain-softening problems, the $\xi_{I}$ and $\xi_{J}$ parameters should be large enough to ensure that localization occurs only at the element ends under discrete load steps. For large load steps and small values of $\xi_{I}$ and $\xi_{J}$, it is possible for these additional integration points to yield simultaneously with the integration points at the element ends. This simultaneous yielding is demonstrated in Figure 10 for relatively small integration parameters $\xi_{I}=\xi_{J}=0.001 L$ and the larger load step values of $\Delta M=0.01 M_{\mathrm{y}}$. It is noted that the likelihood of simultaneous yielding is reduced for more complex constitutive models, e.g. the reinforced concrete fiber sections presented in the following example, where the stiffnesses of the adjacent sections at the element ends differ due to axial-moment interaction. 

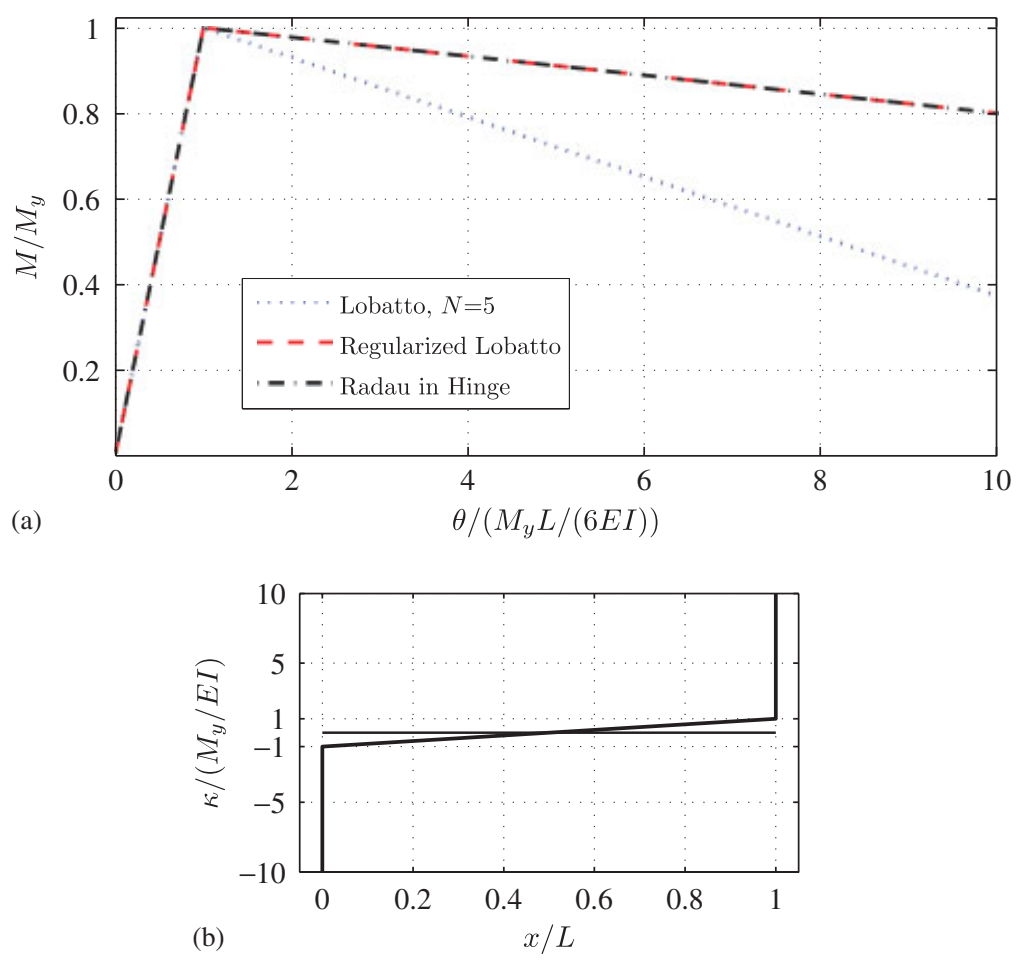

Figure 8. Comparison of beam response between standard and regularized five-point Gauss-Lobatto for strain-softening section behavior: (a) computed moment-rotation response and (b) curvature distribution along the beam.

Based on numerous simulations conducted by the authors, values of $\xi_{I}$ and $\xi_{J}$ equal to $0.1 w_{1}$ and $0.1 w_{N}$, respectively, are ideal for the regularization method to detect the correct behavior for a wide range of constitutive behavior and load increments. The weights $w_{1}$ and $w_{N}$ represent the end weights of the underlying quadrature rule, e.g. for the five-point Gauss-Lobatto rule with end weights $w_{1}=w_{N}=0.05 \mathrm{~L}$, the optimal parameter values are $\xi_{I}=\xi_{J}=0.005 \mathrm{~L}$.

\section{REINFORCED CONCRETE COLUMNS WITH HARDENING AND SOFTENING BEHAVIORS}

To validate the proposed regularization method, the static, cyclic response of two reinforced concrete specimens is simulated. The response of each specimen is computed using a single forcebased element with a regularized five-point Gauss-Lobatto rule with $\xi_{I}=\xi_{J}=0.005 L$. The plastic hinge lengths are determined from the individual specimen properties. The numerical examples are performed in the Open System for Earthquake Engineering Simulation software framework developed as the computational platform for research in performance-based earthquake engineering at the Pacific Earthquake Engineering Research Center [46]. 


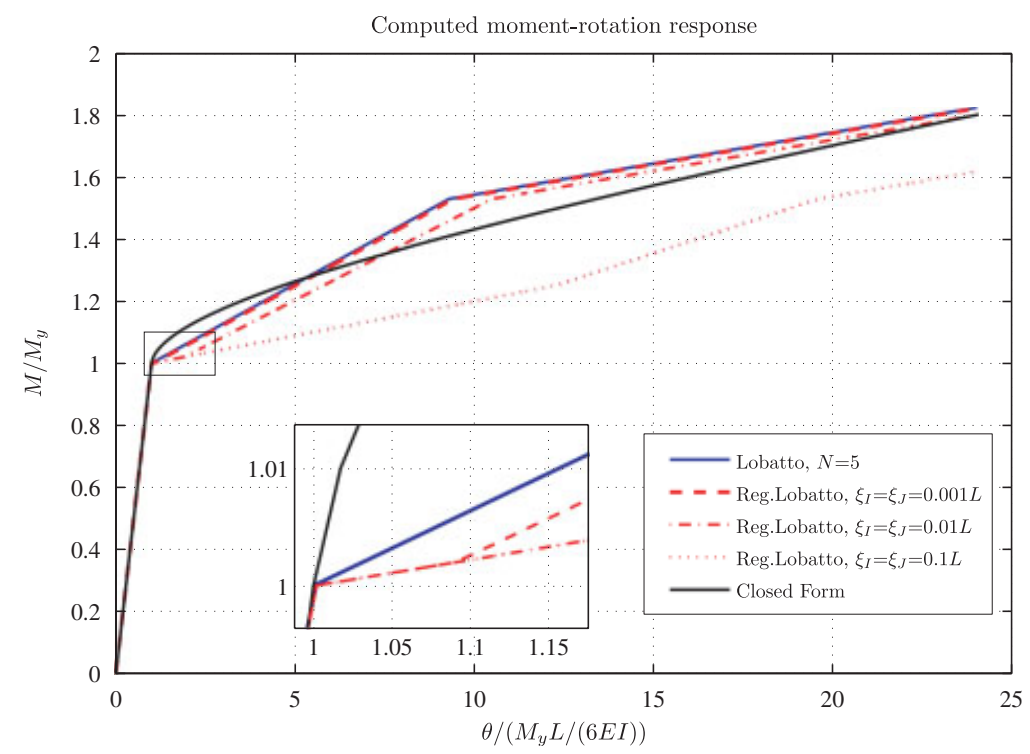

Figure 9. Sensitivity of beam response for increasing values of parameters $\xi_{I}$ and $\xi_{J}$ for regularized five-point Gauss-Lobatto integration and strain-hardening section behavior.

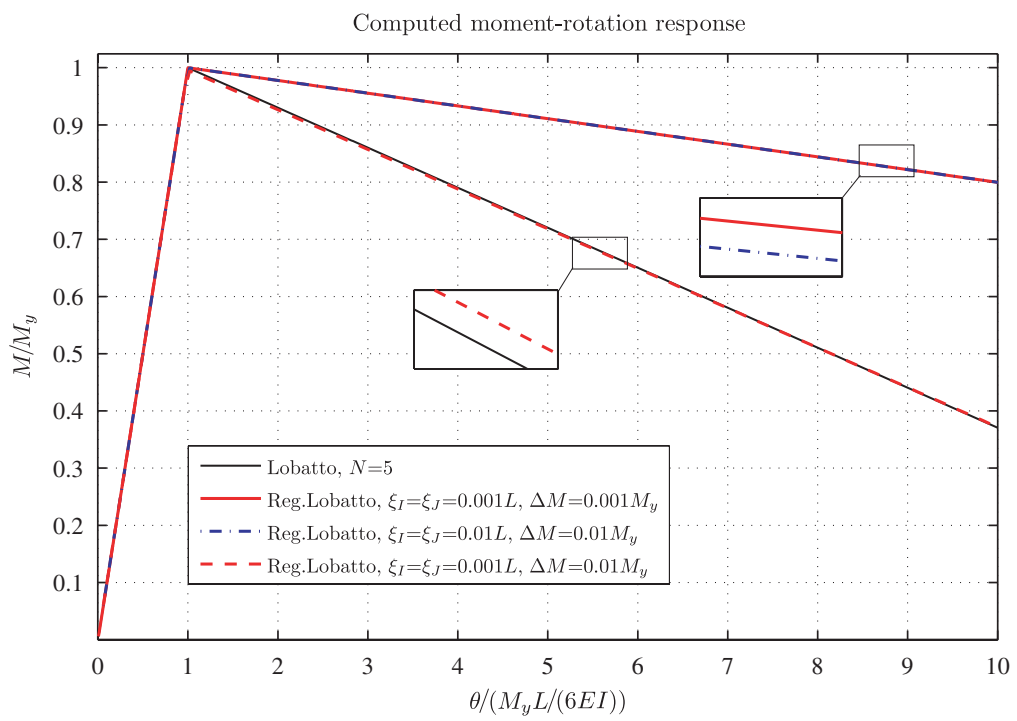

Figure 10. Sensitivity of beam response for various combinations of parameters $\xi_{I}$ and $\xi_{J}$ and load steps for regularized five-point Gauss-Lobatto integration and strain-softening section behavior.

\subsection{Strain-hardening}

A spirally reinforced concrete column, specimen 430 in the tests of Lehman and Moehle [47], is modeled to demonstrate the accuracy of the regularized integration method under strain-hardening 


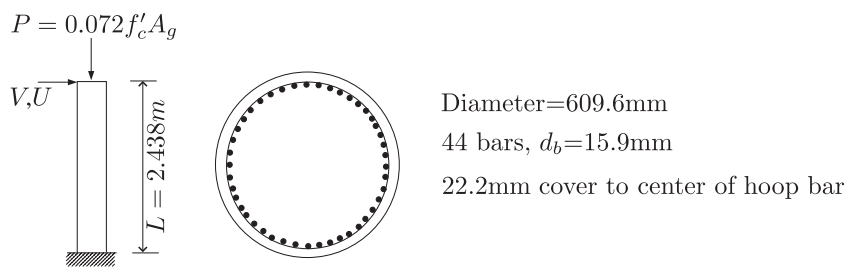

Figure 11. Dimensions of specimen 430 in the test of Lehman and Moehle [47].

behavior. The reinforcing details of the column are shown in Figure 11 and a fiber discretization of the cross section is used to compute the section stress resultants and account for axial-moment interaction. The stress-strain behavior of concrete fibers is modeled by a parabolic ascending branch and linear descending branch in compression [48]. The concrete compressive strength is $f_{\mathrm{c}}^{\prime}=31 \mathrm{MPa}$. Confining effects of transverse reinforcement are estimated using the Mander model [49]. Using this model, the confined concrete has a compressive strength of $f_{\mathrm{cc}}^{\prime}=43.4 \mathrm{MPa}$ reached at a strain of $\varepsilon_{\mathrm{cc}}=0.006$, and ultimate strain $\varepsilon_{\mathrm{ccu}}=0.028$. The reinforcing steel is modeled using the Giuffre-Menegotto-Pinto constitutive model [50]. The elastic modulus, yield stress and hardening ratio of the steel are assumed to be $E=200000 \mathrm{MPa}, f_{\mathrm{y}}=462 \mathrm{MPa}$ and $\alpha=0.01$, respectively. The compressive axial load applied to the specimen is $7.2 \%$ of the axial capacity, $f_{\mathrm{c}}^{\prime} A_{g}$, a relatively light axial load. For the plastic hinge length, an experimentally validated empirical formula that takes into account the effects of bar pullout and strain penetration [51] is used

$$
l_{p}=0.08 L+0.022 f_{\mathrm{y}} d_{\mathrm{b}}(\mathrm{MPa}, \mathrm{mm})
$$

where $L, f_{\mathrm{y}}$ and $d_{\mathrm{b}}$ are the member length, steel yield stress and bar diameter, respectively. Using the column properties, the plastic hinge length is equal to $0.15 \mathrm{~L}$ according to Equation (16). In using this equation to determine the plastic hinge length, it is implicitly assumed that if localization occurs, the region of fracture will be large compared with the individual cracks that contribute to the overall energy dissipation.

The computed load-displacement response of the column is shown in Figure 12(a) and (b) for standard and regularized five-point Gauss-Lobatto integrations, respectively. As seen in the figures, the methods give nearly identical results as yielding spreads from the base of the column under the light axial load. Further evidence of the strong agreement between the standard and regularized integration methods is shown in Figure 12(c) with the moment-curvature response at the base. The results of this example show that the regularized method is able to find a unique solution for strainhardening cyclic response without any spurious behavior arising from the negative integration weight a small distance from the base of the column.

\subsection{Strain-softening}

The reinforced concrete column, specimen BG- 8 in the tests of Saatcioglu and Grira [52], is analyzed in this example. The geometry and reinforcing details of the column are given in Figure 13. As in the previous example, a fiber discretization of the cross section accounts for axial-moment interaction. The same constitutive models for the steel and concrete stress-strain relationships are used in this example. In this case, the concrete compressive strength is $f_{\mathrm{c}}^{\prime}=$ $34 \mathrm{MPa}$. In the core region, $f_{\mathrm{cc}}^{\prime}$ is $49.3 \mathrm{MPa}$ due to the confining effects of the transverse steel. The strain at the ultimate strength and the ultimate strain of core concrete are $\varepsilon_{\mathrm{cc}}=0.007$ and 

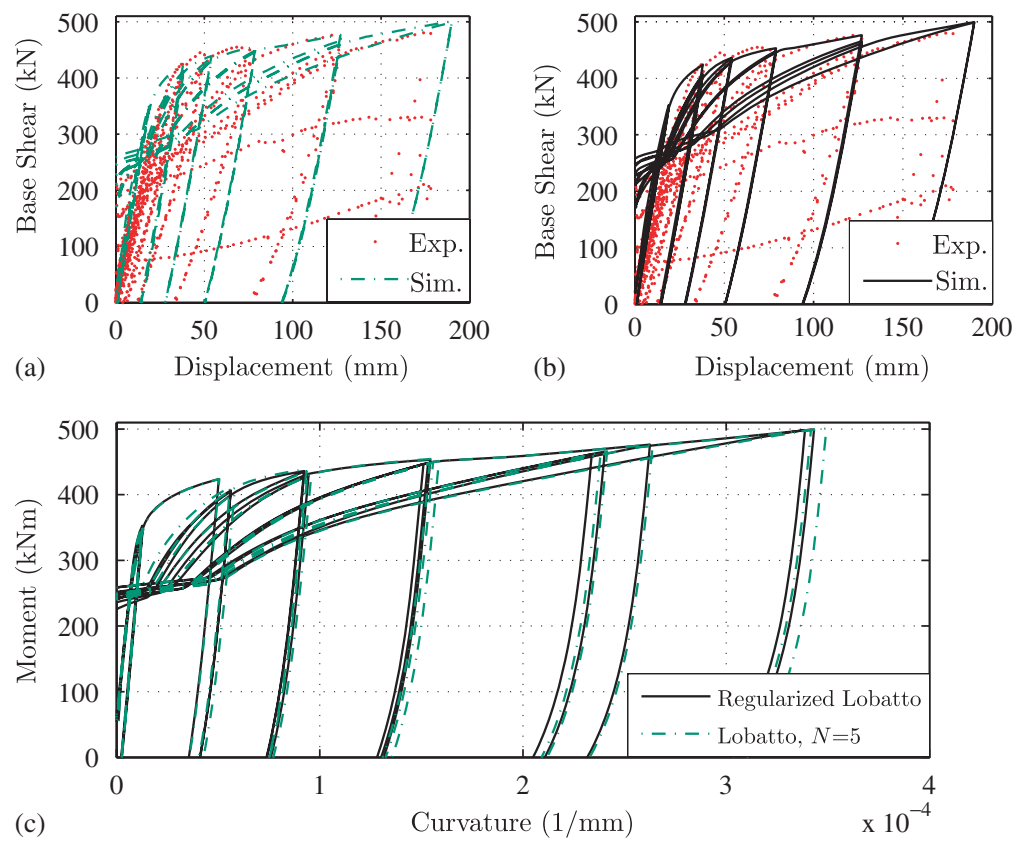

Figure 12. Computed global response and local moment-curvature relationships at the support section of specimen 430: (a) standard Gauss-Lobatto; (b) regularized Gauss-Lobatto; and (c) local response at the support section.
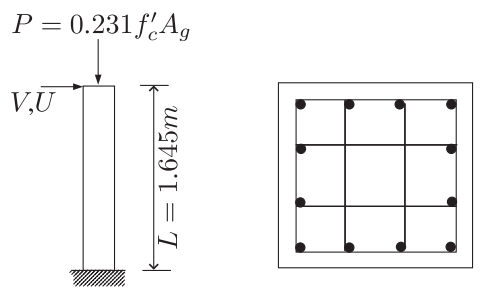

$350 \mathrm{~mm} \times 350 \mathrm{~mm}$

$12 \mathrm{bars}, d_{b}=19.5 \mathrm{~mm}$

$29 \mathrm{~mm}$ clear cover

Figure 13. Dimensions of specimen BG-8 in the tests of Saatcioglu and Grira [52].

$\varepsilon_{\mathrm{ccu}}=0.029$, respectively. For the steel reinforcement, the elastic modulus is $E=200000 \mathrm{MPa}$, yield stress is $f_{\mathrm{y}}=455.6 \mathrm{MPa}$ and hardening ratio is $\alpha=0.01$. A relatively large compressive axial load, $P=0.231 f_{\mathrm{c}}^{\prime} A_{g}$, is applied to the specimen. Using Equation (16), the plastic hinge length is calculated as $l_{p}=0.20 L$.

The global response of the member using standard and regularized five-point Gauss-Lobatto is given in Figure 14(a) and (b), respectively. As seen in the figures, the cyclic response envelope of the cantilever with regularized integration matches the test data for strain-softening behavior. On the other hand, using standard integration, there is a significant discrepancy in the base shear envelope after the plastic hinge forms and damage localizes. Since the numerical solution is obtained by displacement control of the cantilever free end, the computed moment-curvature response in 

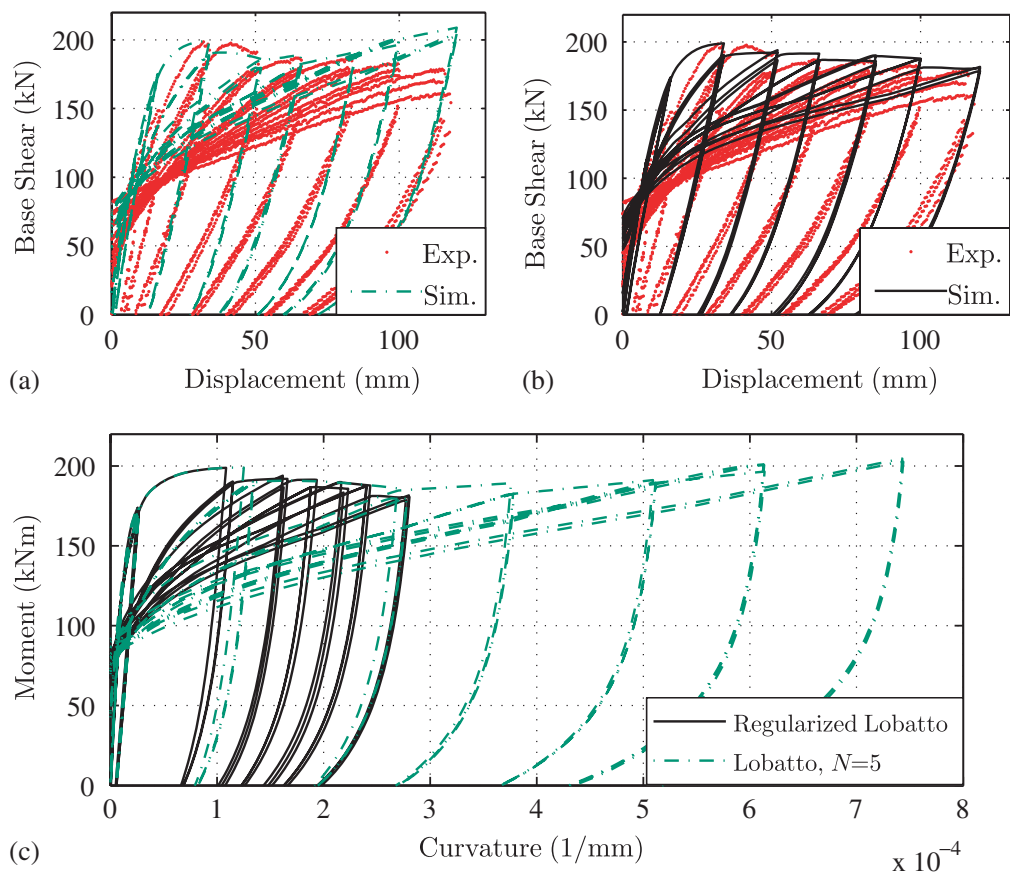

Figure 14. Computed global response and local moment-curvature relationship at the support section of specimen BG-8: (a) standard Gauss-Lobatto; (b) regularized Gauss-Lobatto; and (c) local response at the support section.

Figure 14(c) shows the increased demands imposed at the fixed end as the response localizes over the small plastic hinge length, $0.05 \mathrm{~L}$, implied by the standard five-point Gauss-Lobatto integration. Upon inspection of the computed results, it is noted that the negative integration weight just above the column base does not lead to spurious behavior during cyclic loading with strain-softening section response.

\section{CONCLUSION}

The numerically consistent regularization method developed in this paper mitigates the uncertainty of selecting an integration method to use with force-based frame elements. The integration points at the element ends take on characteristic lengths specified by an analyst in order to regularize localized response in the presence of strain-softening behavior. At the same time, a convergent solution is maintained for the spread of plasticity due to strain-hardening behavior. Interpolatory quadrature ensures that the correct solution for a linear-elastic prismatic element is maintained after the introduction of new integration points and the subsequent manipulation of their weights. The proposed regularization technique is coupled neither to the element constitutive parameters nor to the element state determination algorithm. Furthermore, the examples show that the presence of negative integration weights does not cause erratic numerical behavior under cyclic, static loading. Thus, the proposed method is applicable to any section constitutive model and loading conditions, 
as well as the many variants of force-based element algorithms available in the literature. Future research will focus on analytic sensitivity of the element response with respect to the integration parameters $\xi_{I}$ and $\xi_{J}$ using direct differentiation of the equations that govern the force-based element response [53,54].

\section{ACKNOWLEDGEMENTS}

The regularization method described in this paper was a derivative of research sponsored by the Oregon Department of Transportation (ODOT) into efficient methods of load rating bridge girders. The authors are thankful for the support provided by ODOT; however, the views expressed in this paper do not necessarily reflect those of the sponsor.

\section{REFERENCES}

1. Bažant ZP, Oh BH. Crack band theory for fracture of concrete. Materials and Structures, RILEM 1983; 16: 155-177.

2. de Borst R, Muhlhaus HB. Gradient-dependent plasticity: formulation and algorithmic aspects. International Journal for Numerical Methods in Engineering 1992; 35:521-539.

3. Simo JC, Oliver J, Armero F. An analysis of strong discontinuities induced by strain-softening in rate-independent inelastic solids. Computational Mechanics 1993; 12(5):277-296.

4. Wells GN, Sluys LJ, de Borst R. Simulating the propagation of displacement discontinuities in a regularized strain-softening medium. International Journal for Numerical Methods in Engineering 2002; 53:1235-1256.

5. Bažant ZP, Pan J, Pijaudier-Cabot G. Softening in reinforced concrete beams and frames. Journal of Structural Engineering 1987; 113(12):2333-2347.

6. Bažant ZP, Planas J. Fracture and Size Effect in Concrete and Other Quasibrittle Materials. CRC Press: Boca Raton, FL, 1998.

7. Clough RW, Benuska KL, Wilson EL. Inelastic earthquake response of tall buildings. Third World Conference on Earthquake Engineering, Wellington, New Zealand, 1965.

8. Giberson MF. The response of nonlinear multistory structures subjected to earthquake excitation. Ph.D. Thesis, California Institute of Technology, Pasadena, CA, 1967.

9. Powell GH, Chen PF. 3D beam-column element with generalized plastic hinges. Journal of Engineering Mechanics 1986; 112(7):627-641.

10. El-Tawil S, Deierlein GG. Stress-resultant plasticity for frame structures. Journal of Engineering Mechanics 1998; 124(12):1360-1370.

11. Pincheira JA, Dotiwala FS, D'Souza JT. Seismic analysis of older reinforced concrete columns. Earthquake Spectra 1999; 15(2):245-272.

12. Ibarra LF, Medina RA, Krawinkler H. Hysteretic models that incorporate strength and stiffness deterioration. Earthquake Engineering and Structural Dynamics 2005; 34(12):1489-1511.

13. Jirásek M. Analytical and numerical solutions for frames with softening hinges. Journal of Engineering Mechanics 1997; 123(1):8-14.

14. Marante ME, Picon R, Florez Lopez J. Analysis of localization in frame members with plastic hinges. International Journal of Solids and Structures 2004; 41:3961-3975.

15. Armero F, Ehrlich D. Numerical modeling of softening hinges in thin Euler-Bernoulli beams. Computers and Structures 2006; 84(10-11):641-656.

16. Limkatanyu S, Spacone E. Reinforced concrete frame element with bond interfaces. I: displacement-based, force-based, and mixed formulations. Journal of Structural Engineering 2002; 128(3):346-355.

17. Taylor RL, Filippou FC, Saritas A, Auricchio F. A mixed finite element method for beam and frame problems. Computational Mechanics 2003; 31(1-2):192-203.

18. Alemdar BN, White DW. Displacement, flexibility, and mixed beam-column finite element formulations for distributed plasticity analysis. Journal of Structural Engineering 2005; 131(12):1811-1819.

19. Hjelmstad KD, Taciroglu E. Mixed variational methods for finite element analysis of geometrically non-linear, inelastic Bernoulli-Euler beams. Communications in Numerical Methods in Engineering 2003; 19(10):809-832. 
20. Ciampi V, Carlesimo L. A nonlinear beam element for seismic analysis of structures. Eighth European Conference on Earthquake Engineering, Lisbon, Portugal, 1986.

21. Zeris C, Mahin SA. Analysis of reinforced concrete beam-columns under uniaxial excitation. Journal of Structural Engineering 1988; 114(4):804-820.

22. Spacone E, Ciampi V, Filippou FC. Mixed formulation of nonlinear beam finite element. Computers and Structures 1996; 58(1):71-83.

23. Coleman J, Spacone E. Localization issues in force-based frame elements. Journal of Structural Engineering 2001; 127(11):1257-1265.

24. Addessi D, Ciampi V. A regularized force-based beam element with a damage-plastic section constitutive law. International Journal for Numerical Methods in Engineering 2007; 70(5):610-629.

25. Scott MH, Fenves GL. Plastic hinge integration methods for force-based beam-column elements. Journal of Structural Engineering 2006; 132(2):244-252.

26. Filippou FC, Fenves GL. Methods of analysis for earthquake-resistant structures. In Earthquake Engineering: From Engineering Seismology to Performance-based Engineering, Chapter 6, Bozorgnia Y, Bertero VV (eds). CRC Press: Boca Raton, FL, 2004.

27. Crisfield MA. Non-linear Finite Element Analysis of Solids and Structures, vol. 1. Wiley: New York, 1991.

28. Neuenhofer A, Filippou FC. Evaluation of nonlinear frame finite-element models. Journal of Structural Engineering 1997; 123(7):958-966.

29. Hjelmstad KD, Taciroglu E. Variational basis of nonlinear flexibility methods for structural analysis of frames. Journal of Engineering Mechanics 2005; 131(11):1157-1169.

30. Ranzo G, Petrangeli M. A fibre finite beam element with section shear modelling for seismic analysis of RC structures. Journal of Earthquake Engineering 1998; 2(3):443-473.

31. Schulz M, Filippou FC. Non-linear spatial Timoshenko beam element with curvature interpolation. International Journal for Numerical Methods in Engineering 2001; 50:761-785.

32. Marini A, Spacone E. Analysis of reinforced concrete elements including shear effects. ACI Structural Journal 2006; 103(5):645-655.

33. De Souza RM. Force-based finite element for large displacement inelastic analysis of frames. Ph.D. Thesis, University of California, Berkeley, CA, 2000.

34. Abramowitz M, Stegun CA (eds). Handbook of Mathematical Functions with Formulas, Graphs, and Mathematical Tables (9th edn). Dover: New York, NY, 1972.

35. Taylor RL, Simo JC, Zienkiewicz OC, Chan AC. The patch test: a condition for assessing finite element convergence. International Journal for Numerical Methods in Engineering 1986; 22:39-62.

36. Stoer J, Bulirsch R. Introduction to Numerical Analysis (2nd edn). Springer: New York, NY, 1993.

37. Golub GH, Van Loan CF. Matrix Computations (3rd edn). Johns Hopkins University Press: Baltimore, MD, 1996.

38. Hashemiparast SM, Masjed-Jamei M, Dehghan M. On selection of the best coefficients in interpolatory quadrature rules. Applied Mathematics and Computation 2006; 182:1240-1246.

39. Golub GH, Welsch JH. Calculation of Gauss quadrature rules. Mathematics of Computation 1969; 23:221-230.

40. Gautschi W. On the construction of Gaussian quadrature rules from modified moments. Mathematics of Computation 1970; 24(110):245-260.

41. Booch G, Rumbaugh J, Jacobson I. The Unified Modeling Language User Guide. Addison-Wesley: Reading, MA, 1998.

42. Gamma E, Helm R, Johnson R, Vlissides J. Design Patterns: Elements of Reusable Object-oriented Software. Addison-Wesley: Reading, MA, 1995.

43. Scott MH, Fenves GL, McKenna FT, Filippou FC. Software patterns for nonlinear beam-column models. Journal of Structural Engineering 2008; 134(4):562-571.

44. Petrangeli M, Ciampi V. Equilibrium based iterative solutions for the nonlinear beam problems. International Journal for Numerical Methods in Engineering 1997; 40:423-437.

45. Limkatanyu S, Spacone E. Reinforced concrete frame element with bond interfaces. II: state determinations and numerical validation. Journal of Structural Engineering 2002; 128(3):356-364.

46. McKenna F, Fenves GL, Scott MH. Open System for Earthquake Engineering Simulation. University of California: Berkeley, CA, 2000. Available from: http://opensees.berkeley.edu.

47. Lehman DE, Moehle JP. Seismic performance of well-confined concrete bridge columns. Technical Report PEER 1998/01, Pacific Earthquake Engineering Research Center, Berkeley, CA, 2000.

48. Kent DC, Park R. Flexural members with confined concrete. Journal of the Structural Division (ASCE) 1971; 97(7):1969-1990. 
49. Mander JB, Priestley MJN, Park R. Theoretical stress-strain model for confined concrete. Journal of Structural Engineering 1988; 114(8):1804-1826.

50. Menegotto M, Pinto PE. Method of analysis for cyclically loaded R.C. plane frames including changes in geometry and non-elastic behaviour of elements under combined normal force and bending. Symposium on the Resistance and Ultimate Deformability of Structures Acted on by Well Defined Repeated Loads. International Association for Bridge and Structural Engineering: Zurich, Switzerland, 1973; 15-22.

51. Paulay T, Priestley MJN. Seismic Design of Reinforced Concrete and Masonry Buildings. Wiley: New York, NY, 1992.

52. Saatcioglu M, Grira M. Confinement of reinforced concrete columns with welded reinforcement grids. ACI Structural Journal 1999; 96(1):29-39.

53. Conte JP, Barbato M, Spacone E. Finite element response sensitivity analysis using force-based frame models. International Journal for Numerical Methods in Engineering 2004; 59:1781-1820.

54. Scott MH, Franchin P, Fenves GL, Filippou FC. Response sensitivity for nonlinear beam-column elements. Journal of Structural Engineering 2004; 130(9):1281-1288. 